Y/DZ-1147

Preprint

\section{OAK RIDGE $\mathrm{Y}-12$ PLANT}

MALTUN MARTETRA

\section{INSTRUMENTATION FOR THE DETERMINATION OF \\ MATERIAL PROPERTIES FROM SPECTROSCOPIC MEASUREMENTS OF TOTAL INTEGRATED SCATTER}

\section{G. L. Powell}

T. E. Barber

Oak Ridge Centers for Manufacturing Technologies

J. T. Neu

\section{Surface Optics Corporation i i $30 B \mathbb{E D}$ San Diego, California DEC 191995 OSTI}

Date of Issue: June 19, 1995

\section{Preprint for submission to} the proceedings of SPIE's 40th Annual Meeting and International Symposium on Optical Science, Engineering, and Instrumentation San Diego, Califomia July 9-14, 1995

\section{MANAGED BY}

MARTIN MARIETTA ENERGY SYSTEMS, INC.

FOR THE UNITED STATES

DEPARTMENT OF ENERGY
Prepared by the Oak Ridge Y-12 Plant Oak Ridge, Tennessee 37831 managed by LOCKHEED MARTIN ENERGY SYSTEMS, INC. for the U. S. DEPARTMENT OF ENERGY under contract DE-AC05-84OR21400

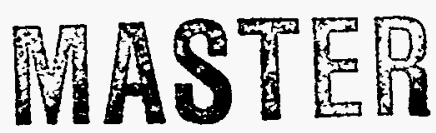




\section{DISCLAIMER}

This report was prepared as an account of work sponsored by an agency of the United States Government. Neither the United States Government nor any agency thereof, nor any of their employees, makes any warranty, express or implied, or assumes any legal liability or responsibility for the accuracy, completeness, or usefulness of any information, apparatus, product, or process disclosed, or represents that its use would not infringe privately owned rights. Reference herein to any specific commercial product, process, or service by trade name, trademark, manufacturer, or otherwise, does not necessarily constitute or imply its endorsement, recommendation, or favoring by the United States Government or any agency thereof. The views and opinions of authors expressed herein do not necessarily state or reflect those of the United States Government or any agency thereof.

COPYRIGHT NOTICE
The submitted manuscript has been anthored by a contractor of the
U.S. Government under contract DE-ACO5-84OR21400.
Accordingly, the U.S. Government retains a paid-up, nonexclusive,
irrevocable, worldwide license to publish or repsoduce the published
form of this contribution, prepare derivative wots, distribute copies
to the public, and perform publicly and display publicly, or allow
others to do so. for U.S. Government purposes.




\title{
Instrumentation for the determination of material properties from spectroscopic measurements of total integrated
} scatter

\author{
G. L. Powell, ${ }^{1}$ T. E. Barber, ${ }^{1}$ J. T. Neu ${ }^{2}$ \\ 'Oak Ridge Centers for Manufacturing Technologies*, Oak Ridge, Tennessee 37831-8096, United States \\ ${ }^{2}$ Surface Optic Corporation, San Diego, California 92131, United States
}

\begin{abstract}
A variety of important optical properties can be determined from spectroscopic analysis of diffuse reflectance of surfaces. The design of a small user friendly, light-weight, field hardened, computer controlled device for performing infrared spectroscopic analysis of trace contaminants on surfaces is described. The device employs a miniature Fourier transform infrared (FTIR) spectrometer with very efficient diffuse reflectance optics and a portable computer to provide reflectance spectra of surfaces measured relative to some idealized surface. These spectra yield qualitative and quantitative chemical information from a host of surfaces that has imminently practical applications in the determination of surface identification, contamination, and degradation.
\end{abstract}

Keywords: surface analysis, surface inspection, infrared, reflectance spectroscopy, diffuse reflectance, stains, materials.

\section{INTRODUCTION}

This paper describes a small user friendly, light-weight, field hardened, computer controlled device for performing infrared spectroscopic analysis of surfaces developed by Surface Optics Corporation, and referred to in this paper as a surface inspection machine-infrared (SIMIR). The SIMIR design is based on a prototype surface inspection instrument, consisting of a Harrick Scientific Barrel Ellipse diffuse reflectance sampling terminal ${ }^{+}$attached to a MIDAC Illuminator Fourier transform infrared (FTIR) spectrometer ${ }^{23}$. In the Surface Optics Corporation SOC 400 SIMIR, the diffuse reflectance optics have been reduced to half the original Barrel Ellipse dimensions, and the spectrometer has been reconfigured to minimize the size and weight. The spectroscopic performance of the total system is optimized for maximum signal-to-noise, which is the key to surface inspection using mid-infrared spectroscopy. This paper also discusses reflectance mid-infrared FTR spectroscopy, and demonstrates its relevance to surface inspection using data obtained from paper, graphite-epoxy laminates ${ }^{2}$, and oil stains on metals ${ }^{3}$.

\subsection{Mid-Infrared FTIR Spectroscopy}

Mid-infrared FTIR spectroscopy is a powerful tool for chemical analysis ${ }^{4}$, being a mature, computer-based technology that has made excellent use of recent progress in computer and software development. An FTIR spectrometer is, typically, a collimated beam of infrared light having the energy range of 400 to $5000 \mathrm{~cm}^{-1}$ (wavelength range of 2 to $25 \mu \mathrm{m}$ approximately corresponding to that of a $1600 \mathrm{~K}$ black body emitter) and modulated by a Michelson Interferometer. This beam is typically' focused through a sample compartment and refocused onto a detector. Optical devices, referred to as accessories, fit inside the sample compartment and manipulate the spectrometer beam to perform specific tasks. The accessories industry is also mature and essential to practical utilization of FTIR spectrometers. The entire optical system is contained in an enclosure that is purged with nitrogen, argon, or air that is free of water and carbon dioxide. Typically FTIR spectrometers have the capability of diverting the collimated beam outside the spectrometer housing where additional accessories (microscopes, chromatographs, etc.) may be attached.

The detector output is collected as a function of the moving mirror position in the interferometer and a number of these "interferograms" are typically co-added to improve signal to noise. The resulting interferogram is converted by a fast Fourier transform to a "single-beam" spectrum representing beam intensity, in arbitrary units, as a function of energy,

*Managed for the U. S. Department of Energy by Lockheed Martin Energy Systems, Inc. under Contract No. DE-AC05-84OR21400. 
usually in units of wavenumbers $\left(\mathrm{cm}^{-1}\right)$. A single-beam spectrum obtained after the light has interacted with a specimen,usually a dielectric material, is ratioed to a single-beam spectrum taken under some idealized condition, usually the absence of the specimen. This ratio represents the light throughput, "transmittance," of the specimen and spectra are typically reported in units of percent transmittance or aborbance. Absorbance units are the negative base-ten logarithm of the transmittance based on the Einstein Absorption Law, i. e., absorption of light passing through a homogeneous film is first-order in distance through the film with the coefficient of this equation being the extinction coefficient. In the simplest of cases, a linear relationship between the extinction coefficient and the concentration of an absorbing specie, known as Beer's Law, forms the starting point for quantitative chemical analysis. In general, the light loss spectrum represented by absorbance units yields a positive, approximately linear, response to the concentrations of absorbing species, and has an easily comprehended mathematical relationship to both the transmittance, the single-beam spectrum, and the reference spectrum. The energy dependence of the extinction coefficient in the mid-infrared region arises from the absorption of light due the vibration of chemical bonds having a dipole. These mid-infrared spectra contain information that directly correlates qualitatively and quantitatively with chemical functional groups. Moreover, mid-infrared spectra have been so extensively used that they can be read like a book by most chemists, and libraries containing spectra for millions of compounds are commercially available.

\subsection{Reflectance Spectroscopy}

The magnitude of the extinction coefficient is such that strong bands in typical pure organic materials totally absorb light over a thickness of approximately $20 \mu \mathrm{m}$. Thus, the role of most accessories is to effectively reduce the areal density of chemical specie in the beam. In contrast, reflectance spectroscopy for surface analysis seeks to exploit this high sensitivity to detect thin dielectric films on metals. The specular reflection of light polarized normal to the plane of a reflective metal surface can obtain spectra of organic ${ }^{3}$ and metal oxide ${ }^{5}$ films with detection limits approaching fractional molecular coverage of the surface, when grazing angles are used to increase the effective path length through the film and light polarization in the plane of the film is eliminated since light of this polarization is not absorbed by the film. Thus, all the light detected carries absorption information and best sensitivities are obtained when the detector sees the maximum light signal in its operational range (i. e., has its maximum signal-to-noise). For films exceeding $1 \mu \mathrm{m}$ in thickness, the mixing of the real and imaginary parts of the refractive index through Fresnel's Law degrades the quality of the spectral data with respect to chemical interpretation. Thus, specular reflectance can be used on mirror-like surfaces for the detection of thin films.

Diffuse reflectance spectroscopy, on the other hand, is based on the simultaneous scattering and absorption of light. This process occurs in dielectric materials such as powders and translucent organic solids such as paper, wood, and coal. Diffuse reflectance occurs in the near-surface region of the bulk material. The process is described in its simplest form by the simultaneous solution of the first-order differential equation for light absorption and the first order differential equation for light scattering. The results is known as the Kubelka-Munk relationship for which the concentration of the absorbing specie is proportional to $(1-R)^{2} / 2 R$, where $R$ is the fraction of light reflected relative to the reference spectrum. This compares to Beer's Law (concentration proportional to $-\log _{20} R$ ). In the limits of low absorbance of light, i. e., as $R$ approaches 1 , the Kubelka-Munk relationship proportionally approaches $(1-R)^{2}$ whereas the Beer's Law relationship proportionally approaches $1-R$ for simple transmission of light. Thus pure diffuse reflectors demonstrate a sensitivity that increases with decreasing concentration of absorber. This increased sensitivity is accompanied by an increased sensitivity to noise and to small differences, often due to instrument instabilities. between the two "single-beam" spectra that are ratioed to produce $\mathrm{R}$. The optics used for diffuse reflectance are designed to reject specularly reflected light and to collect only light scattered by the material of interest.

Diffuse reflectance optics can also be applied to the analysis of films on metal surfaces that have some curvature or surface roughness. The results are essentially specular reflectance measurements obtained by averaging over a wide range of angles of reflectance. The polarization of the light can no longer be controlled by the optics so the specularly reflected light contains polarization components in the plane of the reflecting surface that contribute to the reflectance, not to the absorbance. Both the geometrical effect of the angle of incidence and the unpolarized light reduce the sensitivity of the technique to absorbing species relative to that of grazing-angle specular reflectance measurements. This loss of sensitivity generally translates as more utility of the method for thicker films. As in the case of specular reflectance, very strong absorbers are highly reflective in the region of the strong bands. This decreases the utility of the data for chemical analysis. 
Practical considerations play an important role in any surface inspection process. Most practical surfaces are not mirrors. Often the required spectral information may be no more than patterns sufficient to distinguish between a few distinctly different materials. Quantitative determinations are virtually always based on empirical calibrations. With these concepts in mind, and with the knowledge that reflectance spectra can be obtained with signal-to-noise greater than 5,000 that can be potentially identify millions of chemical species, a SIMIR has been developed that will allow this technology to be brought to bear on real surfaces in practical environments.

\section{THE SIMIR}

The SIMIR shown in Fig. 1 consists of an FTIR spectrometer and a barrel ellipse diffuse reflectance optical system ${ }^{1}$. The diffuse reflectance optics consists of an elliptical mirror of revolution on the inside surface of an aluminum cylinder that has been cut off normal to the major axis inside each focal point (i. e., the "barrel"). The collimated beam from the interferometer enters this ellipse along its minor axis (i. e., the "bung") and is focused by a parabolic mirror, supported through the "bung" and located at the center of the barrel, to a point that is one of the focal points of the ellipse. At the other focal point of the ellipse is the FTIR spectrometer detector. These focal points are separated by 2 in. The common focal point of the ellipse and the parabloid is in the center of and in the plane of a 0.5 -in. circular opening that is the

\section{SOC 400 Surface Inspection Machine INFRARED}

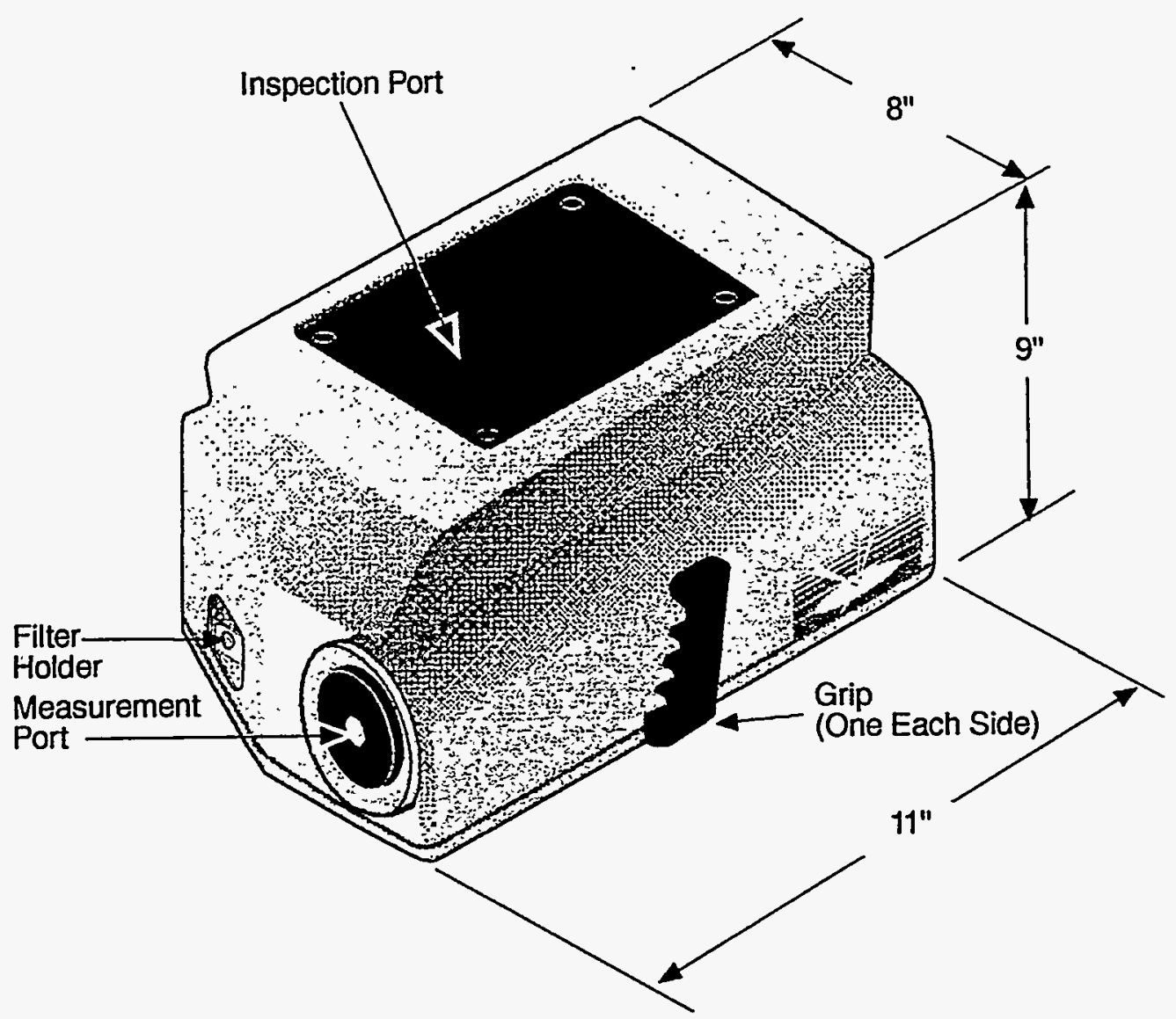

Figure 1. The Surface Optical Corporation Surface Inspection Machine Infrared, SOC 400 SIMIR. 
measurement port. The plane of this circular opening precisely positions the instrument against the surface to be inspected. The hand grips allow the SIMIR to be positioned by hand. Also shown is an inspection port to access the spectrometer optics and electronics, and a filter holder. The filter holder allows a band pass filter to be inserted in the beam that gives an enhanced signal-to-noise over a narrow spectral region when used in concert with a gain enhancement at the detector or increased light from the source. Not shown in Fig. 1 is a power cable up to $25 \mathrm{~m}$ in length supplying the $\sim 40$ watt power requirements of the spectrometer, and a dry and carbon dioxide free gas purge. A portable computer operates the spectrometer via a parallel interface and collects the spectra using MIDAC/GRAMS386 software. The SIMIR shown in Figure 1 weighs under $7 \mathrm{~kg}$ and occupies a $14 \mathrm{~L}$ volume.

The prototype instrument used as the design base for the SIMIR, and used to collect the data described in the remainder of this paper was a MIDAC Illuminator Model 2501-1 mounted to a Harrick Scientific Barrel Ellipse that had a 4-in. focal point separation. The instrument was supported by a $12 \mathrm{VDC}$ power source and a $33 \mathrm{MHz}$, 486DX portable computer operating the instrument using MIDAC/GRAMS386 software. The spectrometer system weighed $13 \mathrm{~kg}$ and had a volume of approximately $25 \mathrm{~L}$. It could be manipulated by hand, served by hand while mounted in a rack, or operate an $X Y$ positioner by RS-232 serial commands issued by the MIDAC/GRAMS386 operating system synchronized with the spectral collection. The positioner allowed spectra from a flat surface to be obtained from a predetermine XY-grid so that surface maps of chemical moieties could be produced. The XY manipulator was a pair of VELMEX UniSlides, each having 350 $\mathrm{mm}$ travel driven by a VELMEX NF90 Controller. The relative positioning error was less than $0.7 \mathrm{~mm} / \mathrm{m}$.

The SIMIR is usually operated at $8 \mathrm{~cm}^{-1}$ resolution, which results in data every $4 \mathrm{~cm}^{-1}$, and zero filled to points every $2 \mathrm{~cm}^{-1}$. Sixty-four scans are coadded to produce a spectrum. Reference spectra for this SIMIR was obtained from a sandblasted gold surface. Sandblasted gold is the most reflective surface found to date for randomly scattering the incident beam. The energy dependence of the reference spectrum from gold is very nearly that of the light beam focused directly on the detector. The gold surface has no chemical features detectable by mid-infrared spectroscopy. The gold surface cleans very easily using common household degreasers such as Formula 409 and distilled water rinse. If that is not sufficient, extremely harsh chemical methods such as concentrated sulfuric or nitric acid may be applied. Being able to clean the reference spectrum is very important since the greatest challenge of surface cleanliness analysis is obtaining confidence that the reference surface is clean. In principle, the reference spectra can be obtained from any surface. Clever choices for reference spectra allow for the direct comparison of the surface of interest to some known surface. So long as the spectra are in absorbance units (i. e., logarithmic units) the reference spectrum can be changed from one surface to another by subtracting one spectrum from another.

\section{APPLICATIONS}

The remainder of this paper describes results obtained by inspecting surfaces with mid-infrared spectroscopy using spectra in absorbance units (a.u.) referenced to clean, sandblasted gold. First, three examples representing very different materials; paper, a graphic-epoxy composite, and oil stains on sandblasted gold; will be presented to demonstrate the data obtained by the SIMIR. Each of these materials represent practical variations in the chemical condition of the surface. The first point to be made is that the spectral patterns are visually obvious. With a little practice, different materials are easily distinguished, as are variations in surface conditions of each of these materials. The second point to be made is that the spectra can be manipulated mathematically and reported as peak heights or areas, calculations based on peak heights or areas, or decisions made from these calculations. The third point is that spectra and calculations from spectra can be made with instrument noise levels of 0.01 to 0.001 a.u. with an update time of $1.5 \mathrm{~s}$. Instrument noise levels can be improved by an order of magnitude by coadding spectra for a minute or two. Spectra from suspect regions can also be averaged and compared with averages over reference regions. This property is extremely useful in the determination of very low levels of surface contamination.

\subsection{Paper}

Paper is to a good approximation a true diffuse reflector with the light scattering/absorption occurring in the near surface region of the bulk paper. Placing a gold mirror underneath a paper sample in no way modifies the spectral features. The examples shown in Fig. 2 are spectra obtained from Post-it note paper. The three spectra overlap for most of the energy range so the upper and lower spectra were offset up or down for ease of comparison. Fig. 2 compares a region of the 


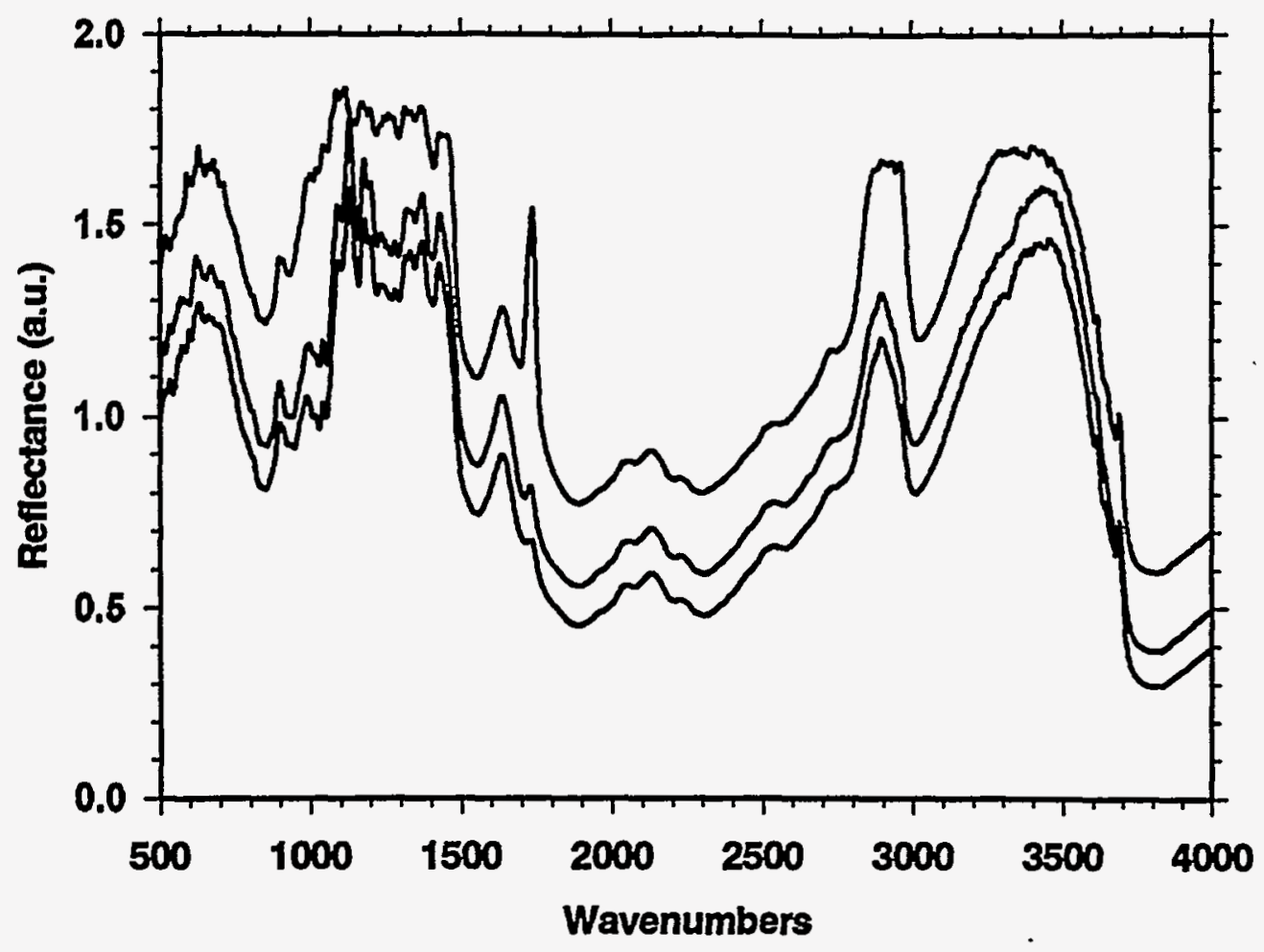

Figure 2. Spectra obtained from the surface of Post-it note paper. Upper spectrum, adhesive side - offset +0.2 a.u.; middle spectrum, side opposite adhesive - offset 0.0 a.u.; lower spectrum, paper not in contact with adhesive - offset -0.1 a.u..

Post-it note that is free of adhesive (lower spectrum) with the region to which adhesive was applied, and to the region from the adjacent page that was in contact with the adhesive (middle spectrum). In the upper spectrum, the applied adhesive is easily detected by the carbonyl band near $1750 \mathrm{~cm}^{-1}$, and by changes in the hydrocarbon band near $2925 \mathrm{~cm}^{-1}$ and the hydroxyl band near $3400 \mathrm{~cm}^{-1}$. The carbonyl band is barely evident in the middle spectrum indicating a small amount of adhesive was transferred to the adjacent note sheet. Subtraction of the lower spectrum from the middle spectrum enhances this feature, and demonstrated that the feature near $1750 \mathrm{~cm}^{-1}$ on the lower curve is distinguishable from the carbonyl band of the adhesive. Other features of these spectra are the sharp bands just below $3700 \mathrm{~cm}^{-1}$, due to alkali earth hydroxides from clays, and the bands between 2000 and $2500 \mathrm{~cm}^{-1}$ that are probably overtones. Bond paper (100\% rag) is very nearly pure cellulose and, except for the broad band near $1650 \mathrm{~cm}^{-1}$, is generally devoid of features between 1500 and $2500 \mathrm{~cm}^{-1}$ and features due to clays. Spectral subtraction of bond paper spectra from these spectra, or the use of bond paper as a reference spectrum, would accentuate features due to additives. The drying of paper by the instrument dry gas purge exhausting through the measurement port has been monitored by observing the $\mathrm{H}-\mathrm{O}-\mathrm{H}$ bending band of molecular water near $1630 \mathrm{~cm}^{-1}$ that is readily apparent from spectra collected over time. Scientific journals such as Physical Review B and Journal of the American Chemical Society have been inspected to determine when (July 1, 1990 and January 1, 1990 respectively) the paper specification was changed to a paper that did not yellow when exposed to light.

\subsection{Graphite-Epoxy Composites}

Graphite-epoxy laminates, and graphite-resin systems in general, form a broad and important class of structural materials known for their high strength and stiffness relative to their weight. They are used extensively in highperformance aircraft. These composites are made from graphite fibers impregnated with partially-cured epoxy, (a tape-like material called a prepreg). Laminate structures are made by layering the prepreg in patterns and then heat curing the laminated prepreg in an autoclave typically near $175^{\circ} \mathrm{C}$. Near or above $175^{\circ} \mathrm{C}$ in air, the mechanical properties of these laminate structures may be degraded as a result of oxidation of the cured resin. The surface of these laminates consists of the outer layer of graphite fiber covered with 10 to $20 \mu \mathrm{m}$ of resin. The incident infrared beam of a SIMIR passes through 
the resin, is effectively scattered by the graphite fiber pattern, and exits through the resin along paths that are appropriate for collection by diffuse reflectance optics.

Figure 3 gives the spectra from Hercules IM6/3501-6 prepreg, as-cured laminate structure, and the structure after being heated in air at $290^{\circ} \mathrm{C}$. Graphite reflects light as a metal with $90 \%$ reflective losses relative to gold as indicated by the baseline level of these spectra being found near 1.0 a.u. The spectrum of the prepreg appears qualitatively similar to the sum of the spectra of the epoxy (tetraglycidyldiaminodiphenylmethane) and the hardener (diaminodiphenylsulphone) except the band near $1650 \mathrm{~cm}^{-1}$, representing the vibrations of two hydrogen atoms on a nitrogen atom of the hardener, is missing and a carbonyl band near $1730 \mathrm{~cm}^{-1}$ has appeared. This is evidence that, during the prepreg preparation, every amine specie in the hardener has lost one hydrogen in the cure reaction and there has been some conversion of the epoxide to a ketone.

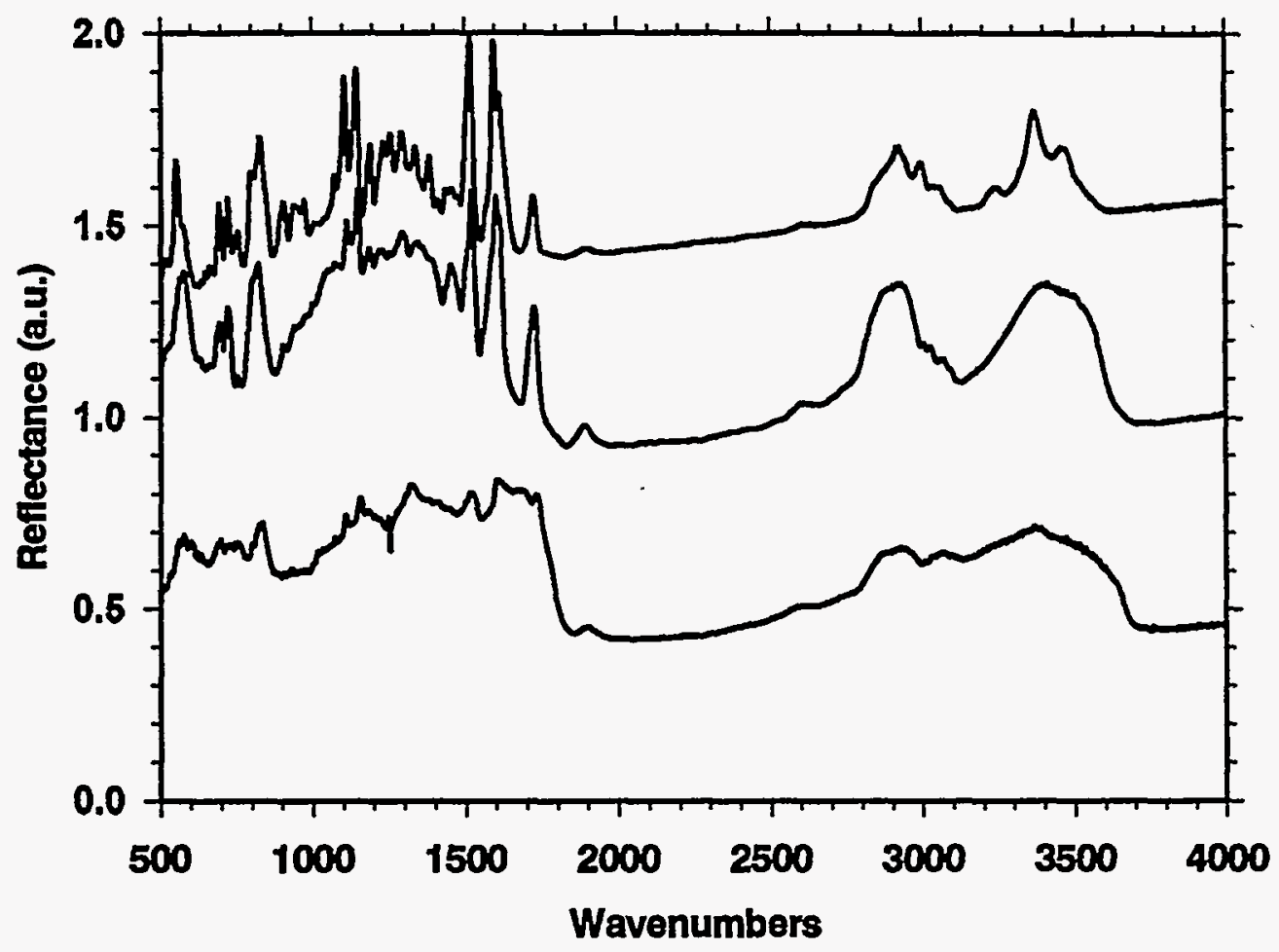

Figure 3. Spectra obtained from the surface of IM6/3501-6 graphite-epoxy composite. Upper spectrum, Prepreg tape partially cured - offset +0.5 a.u.; middle spectrum, heat cured - offset 0.0 a.u.; lower spectrum, oxidized - offset -0.5 a.u..

In the as-cured laminate spectrum many sharp bands have disappeared into broader bands. Many of these bands represent vibrational modes involving many atoms that lose their uniqueness as the resin hardens. The bands that remain distinct, such as those at 1510 and $1600 \mathrm{~cm}^{-1}$, are due to vibrational modes of simple assemblies of atoms relatively unaffected by the cure, in this case the symmetrical breathing motion of benzene rings. The disappearance of bands near $950 \mathrm{~cm}^{-1}$ is due to the epoxide rings being consumed in the reaction. The growth of the band near $3400 \mathrm{~cm}^{-1}$ is the hydroxyl species that is the product of the cure. The loss of the epoxide band and the growth of the hydroxyl band can be correlated during cure. Oxidation results in the formation of carbonyl species $\left(1630\right.$ to $\left.1850 \mathrm{~cm}^{-1}\right)$ due to oxygen addition on its way to becoming carbon dioxide, hydrocarbon loss $\left(2925 \mathrm{~cm}^{-1}\right)$ to form water, and a general loss of the remaining distinct peaks. The oxidized specimen was a flexural strength mechanical test specimen that was oxidized at $288^{\circ} \mathrm{C}$ for 30 min. resulting in a $30 \%$ decrease in load-to-failure. This specimen was one of many covering a broad range of exposure times and temperatures ${ }^{2}$. Reduction of these spectral data to numbers resulted in correlation between spectral data and mechanical test data for which each had comparable statistical validity, i.e., the spectral data could predict the mechanical data with a precision comparable to that of the mechanical data ${ }^{2}$. The distinction between the prepreg, the as-cured laminate, and the 
oxidized laminate is visually obvious and easily reduced to numbers calibrated against other properties. Using a simple 3-point algorithm to monitor the decay of the $1510 \mathrm{~cm}^{-1}$ band, the prototype SIMIR can detect oxidation based on some preset criteria, respond audibly, and display the spectra with an update time of less than $1.5 \mathrm{~s}$.

\subsection{Oil Stains on Gold}

The detection of oil stains on textured metals, to demonstrate surface cleanliness, is another task for which diffuse reflectance optics and the SIMIR are uniquely qualified. Sand blasting is a common preparation for preparing metals for bonding. Certifying the absence of oil contamination that might affect adhesion involves distinguishing types of oil contamination such as vegetable oils, mineral oils, and silicone oils, that may be tolerated at different levels by primers and adhesives, and that may require different cleaning methods for removal. The application of the SIMIR technique to detecting mineral oils and silicone oils on sand blasted aluminum and steel has been described. The main difference between oil detection and the applications discussed so far is that contact between the SIMIR and the substrate should be avoided to prevent oil transferring to the face plate and subsequently onto other surfaces.

Figure 4 compares the spectra of safflower oil, Dow Corning DC $200-5 \mathrm{cpi}$ silicone oil, and pharmaceutical grade mineral oil. Drops of oil were placed on clean sand blasted gold and each drop was vigorously wiped off using clean tissue. This technique leaves a substantial residue behind as a stain representing the upper limit region below which surface inspection for contamination by a SIMIR is desirable. The spectra of the mineral oil consists of bands representing primarily the stretching $\left(2925 \mathrm{~cm}^{-1}\right)$ and bending motions $\left(-1400 \mathrm{~cm}^{-1}\right)$ of the $\mathrm{CH}_{2}$ species. Common automobile oils and greases have similar spectra with some additional structure below $1300 \mathrm{~cm}^{-1}$. The methyl-only bands of the silicone (2965 $\left.\mathrm{cm}^{-1}\right)$ are distinctly different from mineral oil, as are the carbon-silicon bands $\left(1265 \mathrm{~cm}^{-1}\right)$ and the silicon-oxygen bands $\left(\sim 1000 \mathrm{~cm}^{-1}\right)$. The other bands in the silicone spectra are probably additives to lower the viscosity of this heat exchange medium. The safflower oil spectrum resembles the mineral oil spectrum with an additional minor band $\left(3007 \mathrm{~cm}^{-1}\right)$

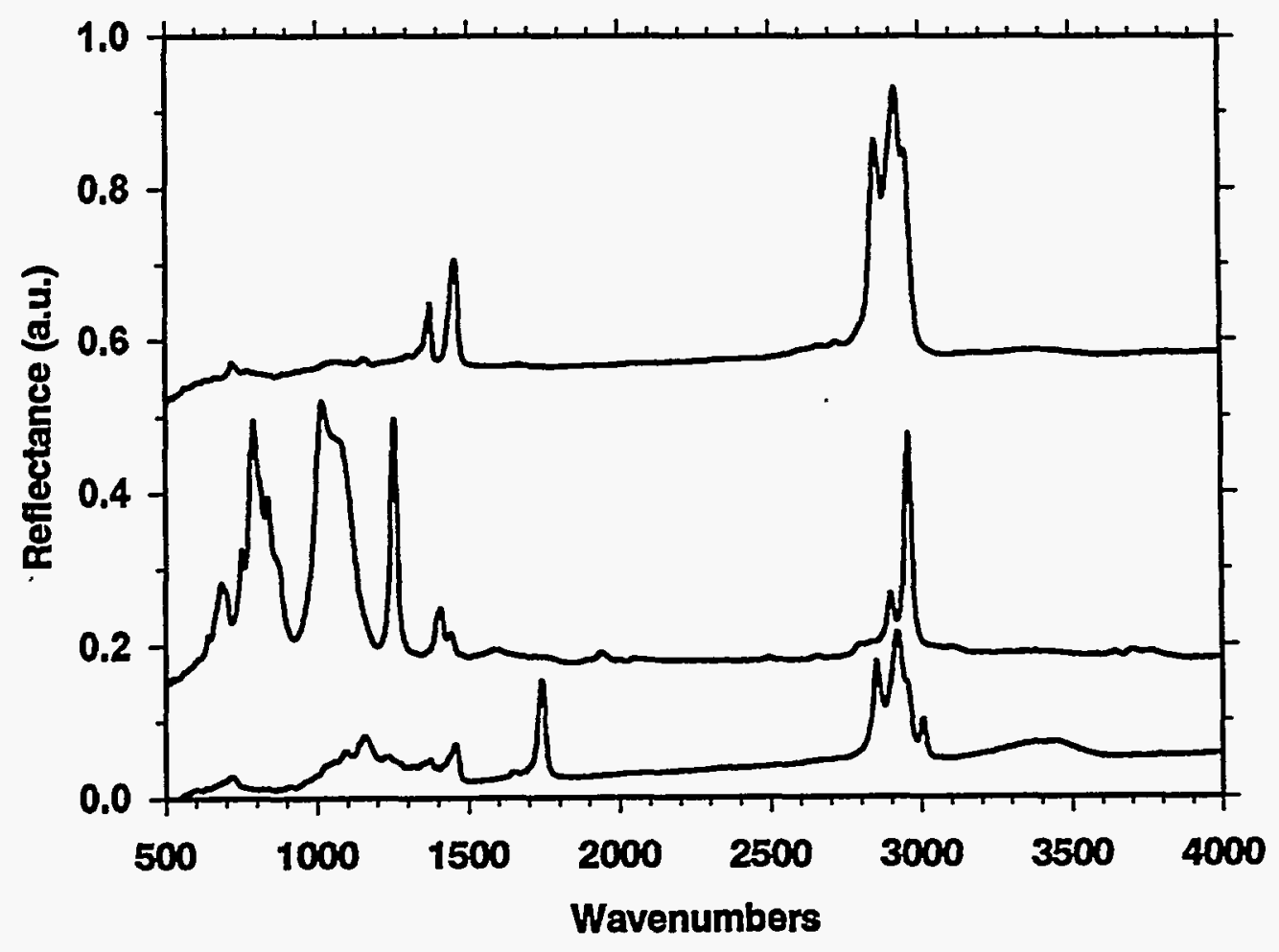

Figure 4. Spectra obtained from the surface of sandblasted gold stained with oil. Upper spectrum, mineral oil - offset +0.2 a.u.; middle spectrum, silicone oil - offset +0.15 a.u.; lower spectrum, safflower oil - offset 0.0 a.u.. 
representing the degree of unsaturation of this cooking oil, and an additional carbonyl band $\left(1735 \mathrm{~cm}^{-1}\right)$ and ester band $\left(1150 \mathrm{~cm}^{-1}\right)$ characteristic of a triglyceride oil. The minor hydroxyl bands $\left(3400 \mathrm{~cm}^{-1}\right)$ are due to water between the oil stain and the gold substrate. These bands are not observed when the oil is applied at low humidities.

\section{SURFACE MAPPING}

The ability to synchronize the position of a flat surface relative to the measurement point of the SIMIR has added a very powerful dimension to the SIMIR. Large numbers of spectra are collected over time from a known grid and packaged as a single file with individual spectra being accessible within that file. Tables of data, such as baseline corrected peak heights, can be produced from thousands of spectra by executing a file that operates on every file in the mult-spectral file. One such program reduces the data to a matrix appropriate for generating surface maps using computer spread sheets. The MIDAC/GRAMS386 operating system and supporting software can also show these spectra as 3-D graphs, contour maps, movies, etc. This section will demonstrate the nature of the SIMIR inspection observations using the XY mapping capability. These observations are related to those results seen by an operator moving the SIMIR about a surface by hand.

\subsection{Paper on Sand Blasted Gold}

The first example was generated by sticking a 12.8 by $12.8 \mathrm{~mm}$ piece of Post-it note paper onto one quadrant of a 75 by $75 \mathrm{~mm}$ clean, sand blasted gold surface. An area 42 by $42 \mathrm{~mm}$ was mapped by moving the measurement point in $1-\mathrm{mm}$ increments in a direction along the diagonal of the paper patch. When 41 spectra had been taken, the $\mathrm{X}$ translator was reset to zero, the $Y$ translator incremented $1 \mathrm{~mm}$, and 41 additional spectra were taken until 1681 spectra were packaged in one file. Figure 5 shows a 3-D plot of 41 such spectra that crossed the paper patch very close to its diagonal. Moving the SIMIR by hand along the same line at a rate of $0.75-\mathrm{mm} \mathrm{s}^{-1}$ (i.e., $40-\mathrm{mm}$ per minute) the operator would see each spectrum displayed in sequence every $1.5 \mathrm{~s}$. The base line noise would be an order of magnitude greater in the fast scan mode, but on the scale of Fig. 5 , it would be barely noticeable. Figure 5 also shows the finite beam size (i.e., finite spatial resolution) of the SIMIR. In a similar experiment the beam was moved linearly across a hair in $0.05-\mathrm{mm}$ steps. From baseline corrected peak height measurements of the hair, the beam was found to be gaussian in shape within $2 \%$ with

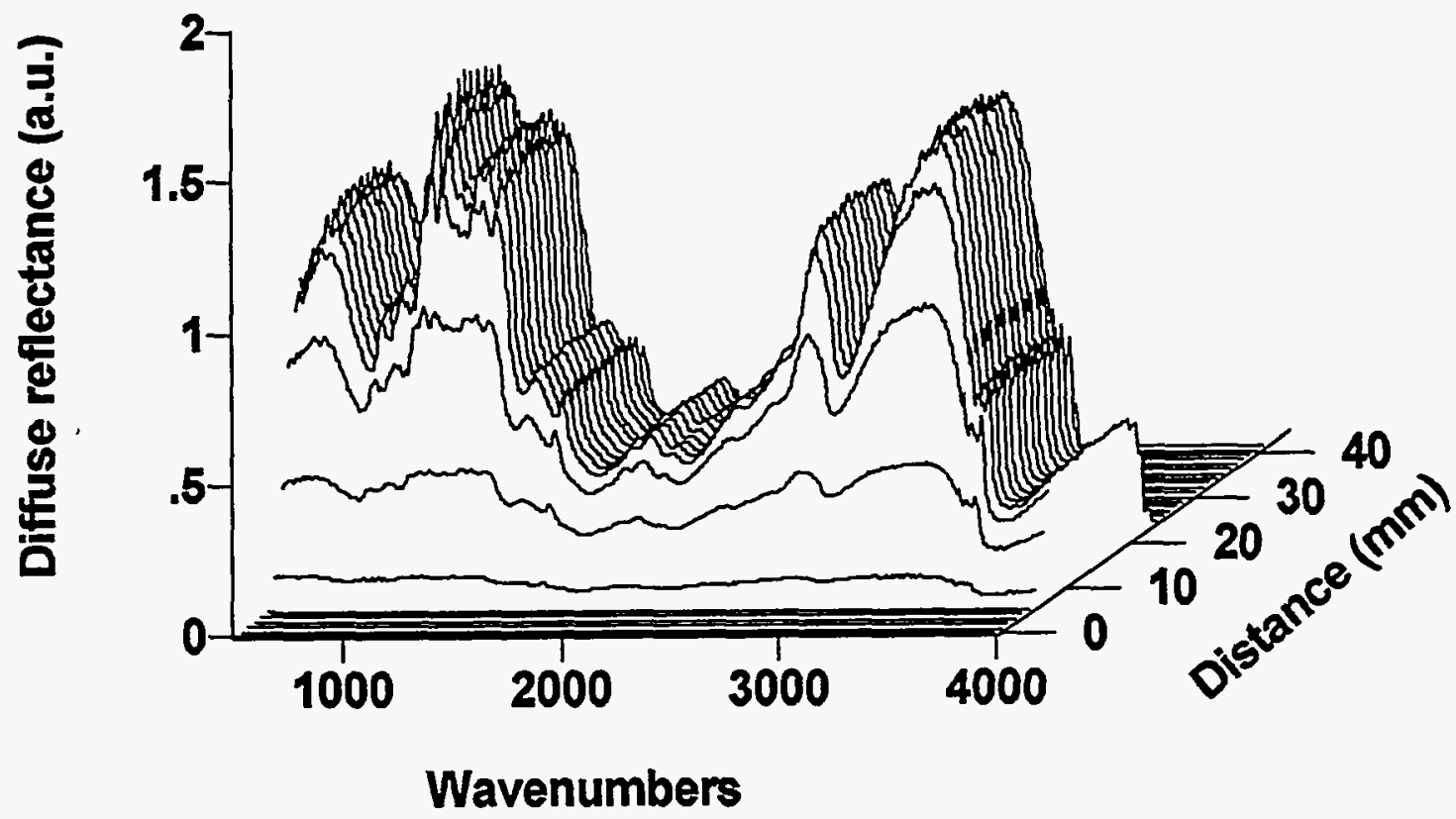

Figure 5. Multiple spectra representing one line of data obtained from the surface of sand blasted gold at $1-\mathrm{mm}$ resolution having a Post-it note patch in one quadrant of the area mapped by the SIMIR. Data collected along the diagonal of the patch. 
a width at half-height of $1.44 \mathrm{~mm}$. In crossing a sharp boundary, the sampling point must travel approximately $4 \mathrm{~mm}$ before evidence of the first side of the boundary disappears. Figure 5 also demonstrates how visually obvious the output of the SIMIR can be.

The data file was processed to determine the baseline corrected peak height of the $\mathrm{CH}_{2}$ stretching band at 2925 $\mathrm{cm}^{-1}$ (i.e., the height of the band was measured relative to a straight line drawn between the values of the spectrum at 2750 $\mathrm{cm}^{-1}$ and at $3050 \mathrm{~cm}^{-1}$. This algorithm is a general purpose technique for detecting the presence of organic matter. Paper has a very distinct $\mathrm{CH}_{2}$ band. The program to reduce the spectra to a table of peak heights used the map grid to output the results in a matrix format suitable for spread sheet surface map graphics. Figure 6 shows the resulting surface map. Note that the shape of the paper patch, the finite spatial resolution of the SIMIR, and the flat region of the clean gold are obvious. What is not obvious is that the clean gold surface averaged over 1130 spectra, from the 3 quadrants of the surface that had no paper, yielded an average value of -0.00014 a.u. \pm 0.00040 Students $t 95 \%$ confidence limits for the next measurement (i.e., the mapping was done with an elevation resolution of 1 part per 1000 per measurement). Figure 7 further describes the SIMIR's potential for measuring surface cleanliness by comparing the noise level in the $\mathrm{CH}_{2}$ stretch region to the $\mathrm{CH}_{2}$ pattern, taken from Fig. 4, that is to be detected. This agrees well with the error limits for the peak height algorithm. Figure 7 also shows the versatility of mapping operations in that 20 spectra from one region were averaged and compared with the average of 861 spectra from another region of the gold surface. This significantly decreases the noise level and shows that the 20 spectra region had more $\mathrm{CH}_{2}$ than the average of the larger region by $0.0002 \mathrm{a}$.u. The presence of both $\mathrm{CH}_{2}$ peaks confirms this conclusion. The multi-spectral files obtained by spatial mapping can be manipulated so that areas thought to contain contamination can be averaged and compared to areas thought to be clean with tremendous improvements in the detection limit.
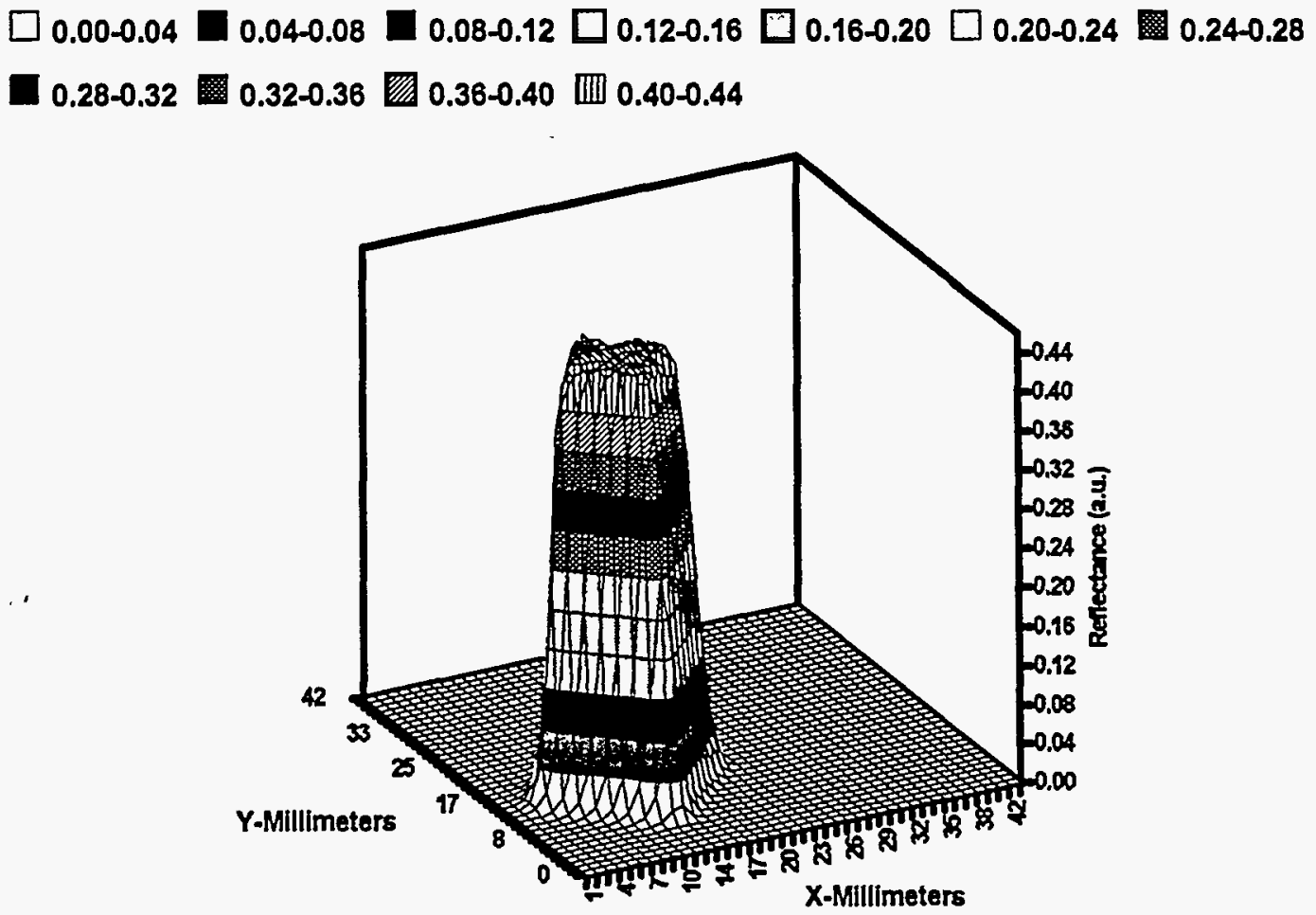

Figure 6. Surface map determined from the baseline corrected $\mathrm{CH}_{2}\left(2925 \mathrm{~cm}^{-1}\right)$ peak height determined for a 40 by $40 \mathrm{~mm}$ area with $1 \mathrm{~mm}$ resolution having a Post-it note patch (12.8 by $12.8 \mathrm{~mm}$ ) in one quadrant. 


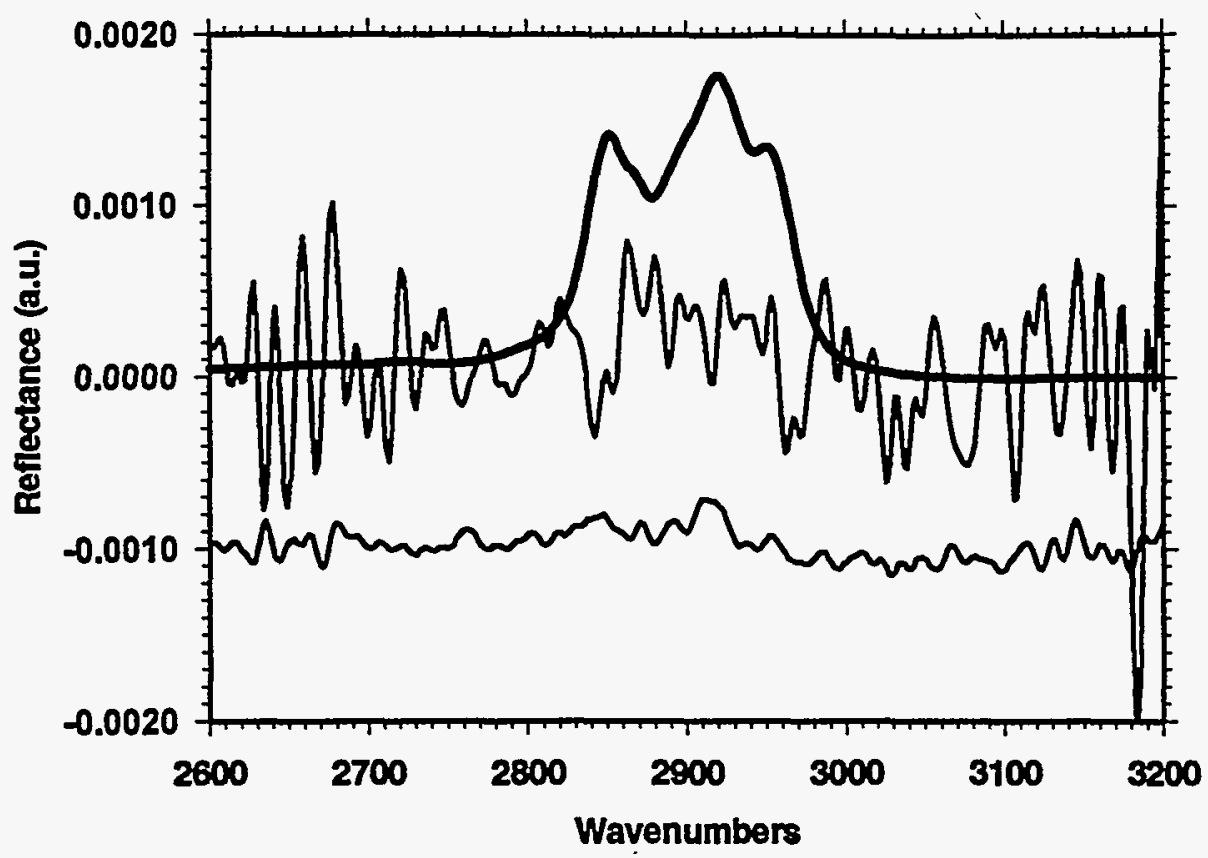

Figure 7. Noise limitations determined from the baseline corrected $\mathrm{CH}_{2}$ stretch near $2925 \mathrm{~cm}^{-1}$. The bold curve is the pattern to be detected overlaid on typical spectral noise. Lower curve shows noise improvements due to averaging.

\subsection{Safflower Oil on Sand Blasted Gold}

A $1 \mu \mathrm{L}(0.92 \mu \mathrm{g})$ drop of Safflower oil was placed on a clean gold substrate similar to that used in the paper experiment. The drop was dispensed using a precision microliter syringe. The drop slowly spread over a time interval of weeks to a diameter of approximately $40 \mathrm{~mm}$. The area was mapped on a 30 by 30 grid with $2-\mathrm{mm}$ spatial resolution and $16 \mathrm{~cm}^{-1}$ spectral resolution without zero filling to minimize the multi-spectral file size. Spectra obtained along a line across the center of the spot is shown in Figure 8. The spectra are clearly recognizable as a triglyceride and clearly distinguishable from mineral oil and silicone oil. The oil spreads with a visual sharp boundary and mapping confirmed the definition of that boundary. The sloping baseline of the spectra where oil is present are reflective losses that, when extrapolated to higher energies in the visible range, results in the stain being visible. Following the locus of the baseline at $4000 \mathrm{~cm}^{-1}$ indicates that some discontinuities exist in the reflectivity of the substrate that Figure 9 will show to have little effect on the $\mathrm{CH}_{2}$ baseline corrected peak height measurement.

Figure 9. gives the surface map based on the same algorithm used to analyze the paper on sand blasted gold described in 4.1. The oil had spread very uniformly over the gold substrate and established uniformly steep concentration gradients at the edge of the stain. The number matrix used to plot Figure 9 was numerically integrated over the entire surface to yield a value that, when divided into the weight of the oil, yielded a calibration factor for converting absorbance units into weight per unit area. The calibration factor was determined to be $6.0 \mathrm{~g} \mathrm{a.u.}{ }^{-1} \mathrm{~m}^{-2}$. A similar experiment using a pharmaceutical grade mineral oil drop resulted in a larger and more irregular stain, probably a result of fractionation of the molecular species that comprise the oil. Thus, the maximum thickness of the film shown in Figure 9 was $1.1 \mathrm{~g} \mathrm{~m}^{-2}$ or 1.2 $\mu \mathrm{m}$ film thickness. The trace of oil shown in the lower spectrum in Figure 7 would represent $1.3 \mathrm{~nm}$ film if this calibration extrapolates linearly as the film thickness approaches zero. Earlier experiments on aluminum and steel indicate that there is a decrease in sensitivity at low film thickness, and that the calibration factor is a strong function of the substrate ${ }^{3}$. Whether this substrate dependence is a function of the surface texture or the substrate material has not been determined. 


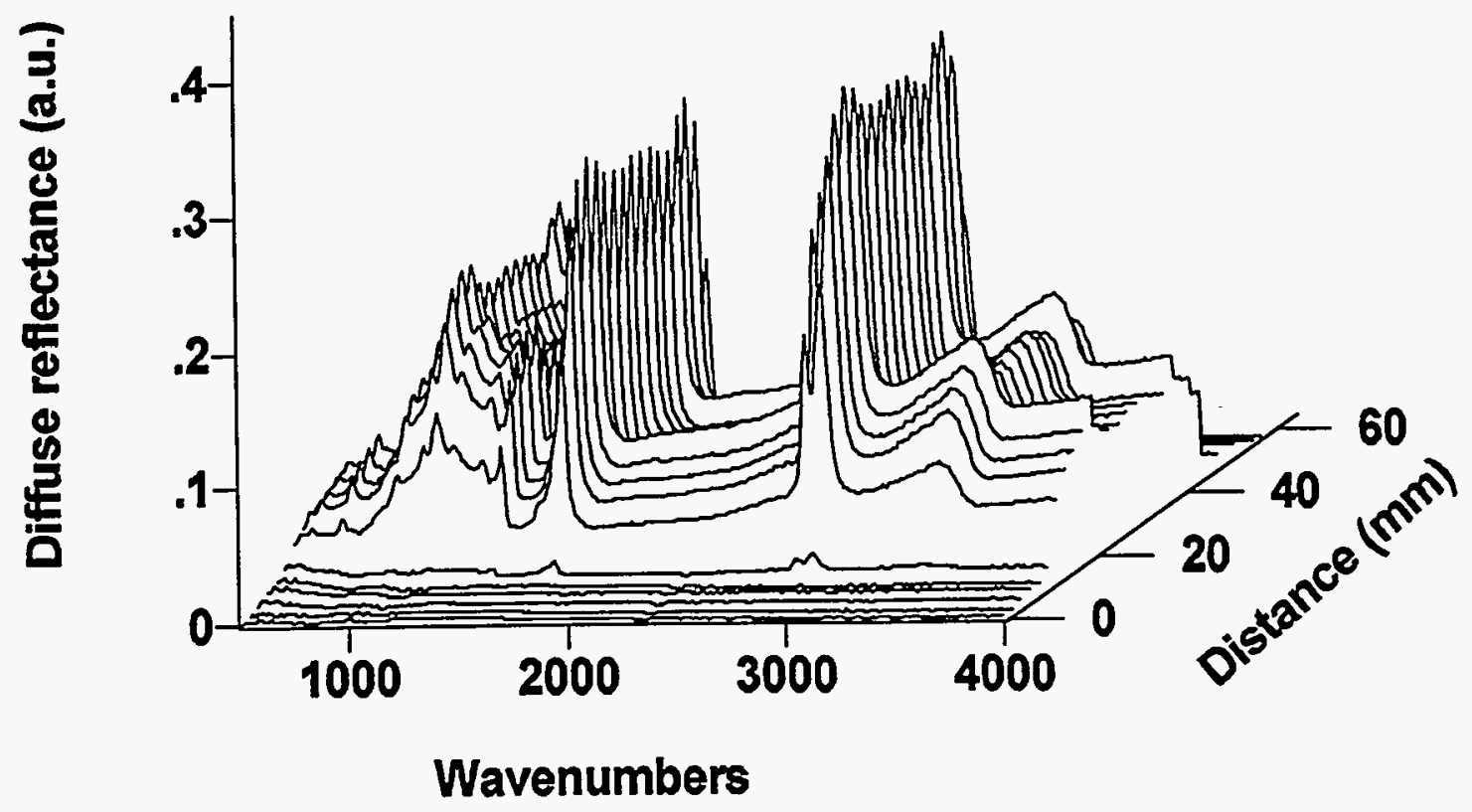

Figure 8. Multiple spectra representing one line of data obtained from the surface of sand blasted gold after having placed a $1 \mu \mathrm{L}$ drop of safflower oil near the center of the 58 by $58 \mathrm{~mm}$ area mapped by the SIMIR.
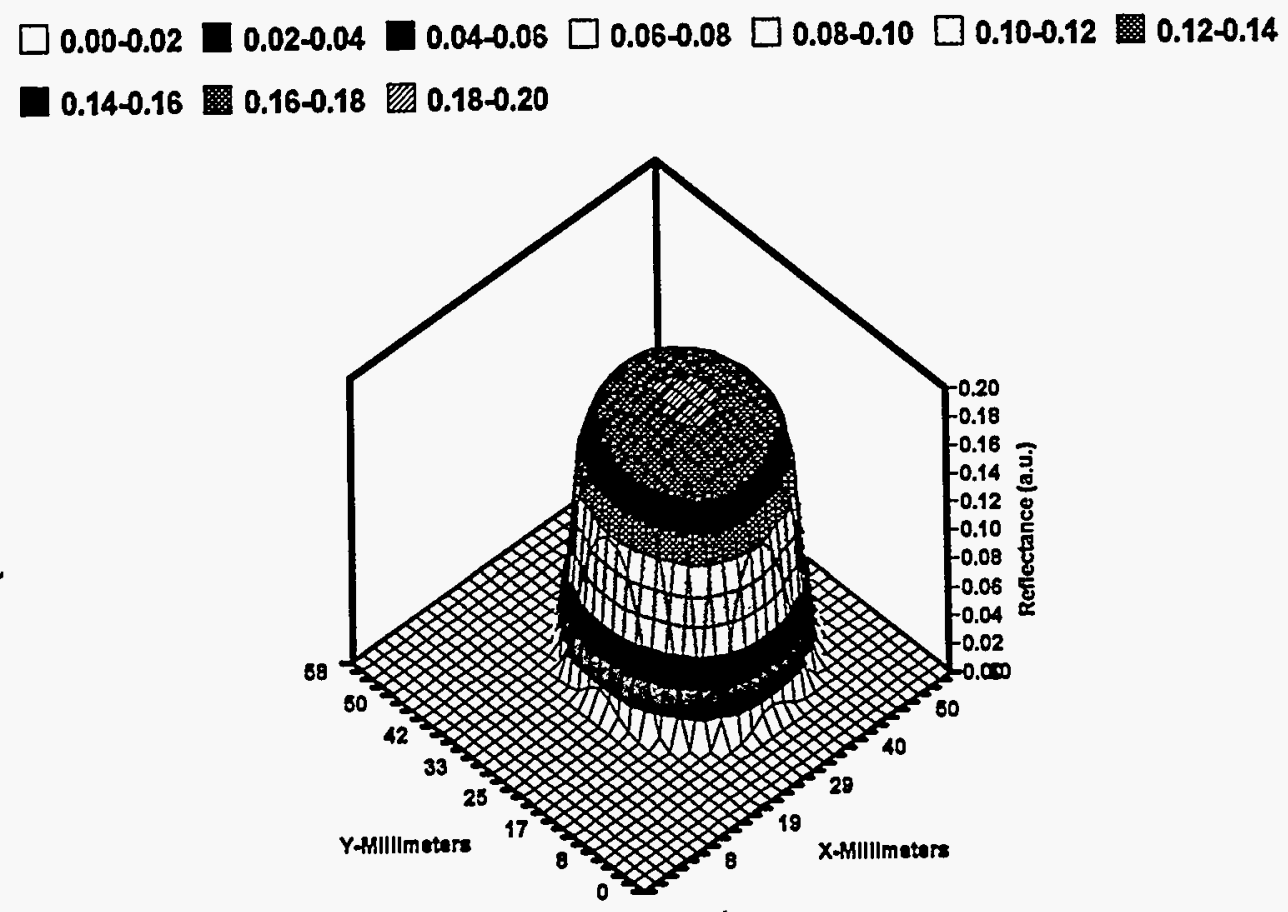

Figure 9. Surface map from the $\mathrm{CH}_{2}$ stretch $\left(2925 \mathrm{~cm}^{-1}\right)$ determined for a 58 by 58 $\mathrm{mm}$ area with $2 \mathrm{~mm}$ resolution due to a $1 \mu \mathrm{L}$ drop of safflower oil migrating for 3 weeks prior to mapping by the SIMIR. 


\section{CONCLUSIONS}

The surface inspection machine, infrared (SIMIR) is a powerful tool that has applications to many diverse and important problems. It is a long needed tool for qualitatively and quantitatively determining surface cleanliness, degradation, and other surface and near surface properties. For film in the thickness range of $100 \mathrm{~nm}$ to $100 \mu \mathrm{m}$ the SIMIR has a tremendous range of chemical information at its disposal. The dynamic range of this prototype SIMIR is sufficiently large that detection of surface contamination in the nanometer film thickness range is a real possibility. Laboratory spectrometers performing similar tasks have an order of magnitude lower noise level than the prototype SIMIR used here. One goal of the SOC 400 SIMIR is to improve the noise level to a range comparable to the best laboratory instruments. The technology on which the SIMIR is based has a vast array of options with which the instrument can be adapted to a particular task. The most promising such option is that if the noise level is suppressed below the needs of the user, then that excess sensitivity can be directly converted to speed of operation. There are a number of applications that involve little more than the simple identification of a material based on matching one of a few patterns. There are other applications that require unraveling the puzzle of complex chemical mixtures of unknown chemicals. In either case the SIMIR provides the tools to execute the measurement and to challenge the scientist/engineer to solve the puzzle, and, once solved, to train the SIMIR to solve that problem next time.

\section{REFERENCES}

1. G. L. Powell, M. Milosevic, J.Lucania, and N. J. Harrick, "The Spectropus System, Remote Sampling Accessories for Reflectance, Emission, and Transmission Analysis using Fourier Transform Infrared Spectroscopy," Appl. Spectrosc. 46, 111 - 125 (1992).

2. G. L. Powell; N. R. Smyrl, C. J. Janke, E. A. Wachter, W. G., Fisher, J. Lucania, M. Milosevic, and G. Auth, "Nondestructive Inspection of Graphite-Epoxy Laminates for Heat Damage using DRIFT and LPF Spectroscopies," in The Proceedings of the Conference on Characterization and NDE of Heat Damage in Graphite Epoxy Composites, NTLAC, Austin, Texas, 97 - 111 (1993).

3. G. L. Powell, N. R. Smyrl, D. M. Williams, H. M. Meyers, III, T. E. Barber, M. Marerro-Rivera, "Surface Inspection Using Fourier Transform Spectroscopy," Aerospace Environmental Technology Conference, NASA Conference Publication 3298, A. F. Whitaker, ed., MFSC, Alabama, 563-571 (1995).

4. P. R. Griffiths, and J. A. DeHaseth, Chemical Infrared Fourier Transform Spectroscopy, (Wiley, New York, 1986)

5. G. L. Powell, A. G. Dobbins, S. S. Cristy, T. L. Clif, H. M. Meyers, J. Lucania, M. Milosevic, "The Study of the Oxidation of Uranium by External and Diffuse Reflectance FTIR Spectroscopy using Remote-Sensing and Evacuable Cell Techniques," SPIE--The International Society for Optical Engineering Proceedings, 2089, 214-215 (1993) 


\section{Distribution}

SPIE's 40th Annual Meeting and International Symposium on Optical Science. Engineering, and Instrumentation

SPIE Proceedings

John C. Stover

Surface Optic Corporation

J. T. Neu

\section{Qak Ridge Y-12 Plant}

T. E. Barber

A. K. Lee/DOE-OSTI (2)

G. L. Powell

K. F. Simon/W. E. Smith

Y-12 Central Files - (RC) 
